\title{
Cardiac Diastolic Function Is Impaired at Rest and Worsens With Exercise in Otherwise Healthy Individuals With Insulin Resistance
}

\author{
Caglar Emre Cagliyan,,${ }^{1}$ MD, Sinan Kirim, ${ }^{2}$ MD, Serdar Turkmen, ${ }^{3}$ MD, Kamuran Tekin,,${ }^{4}$ MD, \\ Mehmet Balli, ${ }^{4} \mathrm{MD}$, Levent Ayman, ${ }^{2} \mathrm{MD}$, Rabia Eker AKILli, ${ }^{1} \mathrm{MD}$, \\ Arafat YILDIRIm, ${ }^{4} \mathrm{MD}$, and Murat CAYLI, ${ }^{4} \mathrm{MD}$
}

\begin{abstract}
SUMMARY
Insulin resistance (IR) is a pathophysiological condition and is associated with cardiovascular risk factors including heart failure. However, studies demonstrating myocardial abnormalities in the early phases of IR are limited. The aim of this study was to investigate myocardial function in otherwise healthy individuals with IR.

Individuals with IR who were free of cardiovascular risk factors and healthy controls were included. Stress echocardiography with tissue Doppler imaging (TDI) was performed. Systolic and diastolic TDI waves were compared in both groups.

A total of 77 individuals (51 with IR and 26 controls) were included in our study. The tissue early flow (e')/atrial contraction (a') ratio at rest was significantly lower in the IR group $(P=0.003)$. The annular early flow $(\mathrm{E}) / \mathrm{e}^{\prime}$ ratio, a predictor of left ventricular filling pressure, was similar in both groups at rest $(P=0.522)$. After exercise, e'/a' impairment became more prominent in the IR group $(P<0.001)$; whereas the E/e' ratio was also significantly lower $(7.6 \pm 1.8$ versus $6.7 \pm 0.9 ; P=0.007$ ) in the IR group.

Myocardial involvement seems to occur in patients with IR, before the appearance of other cardiovascular risk factors. Exercise induced diastolic worsening may be a predictor of reduced compliance and increased ventricular stiffness. More detailed prospective studies are required for more precise results. (Int Heart J 2015; 56: 345-348)
\end{abstract}

Key words: Diastolic stress echocardiography, Heart failure, Tissue Doppler

I nsulin resistance (IR) is a pathophysiological condition in which normal insulin concentrations cannot produce an adequate insulin response in peripheral target tissues such as the liver, muscle, and adipose. This is compensated for by the excessive secretion of insulin by pancreatic beta cells resulting in hyperinsulinemia. ${ }^{1)}$ The resistance in these tissues coupled with the increased effects of hyperinsulinemia in the sensitive tissues leads to metabolic syndrome (MetS), ${ }^{2)}$ and MetS is a very strong predictor of new-onset Type-2 diabetes mellitus (T2DM). ${ }^{3)}$ Metabolic syndrome causes a 2-fold increase in cardiovascular outcomes. ${ }^{3,4)}$ Insulin resistance is associated with increased left ventricular mass and this increase becomes more apparent with worsening glucose tolerance. ${ }^{5)}$

Individuals with IR are at increased risk of cardiovascular adverse outcomes, including heart failure (HF). Metabolic disorders associated with IR usually precede and contribute to HF genesis. ${ }^{6}$ Also, hypertension (HT) is another major risk factor strongly correlated with IR, left ventricular hypertrophy, and $\mathrm{HF}^{7,8)}$ However, some papers have found IR is an independent predictor of HF, regardless of other known risk factors. ${ }^{9,10)}$ On the other hand, HF itself leads to the genesis and progression of IR via several mechanisms. ${ }^{11)}$ Hence, it is not untrue to state that there is a chicken and egg situation between IR and HF.

Although there is a strong relationship between IR and $\mathrm{HF}$, there is limited data about the exact mechanisms contributing to this pathophysiologic process. The aim of this study was to identify myocardial abnormalities at rest and post exercise in otherwise healthy individuals with IR who did not fulfill MetS criteria.

\section{MeTHODS}

After being approved by the local ethics committee, our study was conducted at Adana Numune Training and Research Hospital (ANTRH). The study was conducted in accordance with the Declaration of Helsinki.

Patient selection: Patients admitted to the ANTRH Endocrinology Outpatient Clinic were enrolled after providing written informed consent. Patients with insulin resistance and

From the ${ }^{1}$ Department of Cardiology, Cukurova University Faculty of Medicine, ${ }^{2}$ Department of Internal Medicine, Adana Numune Training and Research Hospital, Adana, ${ }^{3}$ Department of Cardiology, Sanko University, Gaziantep, and ${ }^{4}$ Department of Cardiology, Adana Numune Training and Research Hospital, Adana, Turkey.

Address for correspondence: Caglar Emre Cagliyan, MD, Department of Cardiology, Cukurova University Faculty of Medicine, 01330, Balcali, Saricam, Adana, Turkey. E-mail: cemrec76@hotmail.com

Received for publication August 1, 2014. Revised and accepted October 27, 2014.

Released in advance online on J-STAGE April 23, 2015.

All rights reserved by the International Heart Journal Association. 
without any other cardiovascular risk factor including MetS were included. Exclusion criteria are listed in Supplemental Table I. The control group consisted of healthy obese volunteers without insulin resistance.

Laboratory tests: Serum glucose, insulin, low-density lipoprotein (LDL)-cholesterol, high-density (HDL) lipoprotein, and triglyceride levels were studied after a 12-hour fasting period in all individuals.

Assessment of insulin resistance: The IR state was determined using the homeostasis model assessment-insulin resistance (HOMA-IR) index. The HOMA-IR index was calculated according to the formula: [Fasting glucose $(\mathrm{mg} / \mathrm{dL}) \times$ Fasting insulin $(\mathrm{uIU} / \mathrm{mL})] \div 405$. Patients with a HOMA-IR index $>2.60$ were judged to have IR. ${ }^{12)}$

Echocardiographic examination: Echocardiographic examination was performed with a GE Vingmed US System Vivid 7 Echocardiograph with the patient laying down in the left lateral decubitus position. Echocardiographic and Doppler images were recorded in the database, and measurements were performed after completion of the study by an experienced echocardiographer. Recorded images of a patient at rest and post-exercise are shown in Supplemental Figure 1. Left ventricular mass $\left(\mathrm{LV}_{\text {mass }}\right)$ was calculated in all individuals, after taking measurements of the left ventricle in the parasternal long axis plane. Left ventricular mass was divided by body surface area to obtain the left ventricular mass index (LVMI). ${ }^{13)}$

All of the Doppler measurements were performed at expiration at a sweep speed of $50 \mathrm{~mm} / \mathrm{second}$. Mean values of 3 consecutive beats were taken into consideration. All of the measurements were performed twice, and the intraobserver variability (IOV) of the mean values was calculated. Cardiac inflow velocities were obtained by pulsed wave (PW) Doppler analysis performed in the apical-4 chamber (A4C) plane. Peak early filling (E) and late atrial contraction (A) wave velocities were measured; the proportion of E/A waves was calculated. Mitral annular velocities were measured in the $\mathrm{A} 4 \mathrm{C}$ plane at the septal and lateral mitral leaflet insertion sites using PW tissue Doppler imaging (TDI). Tissue Doppler PW tracings were recorded at a sweep rate of $66.7 \mathrm{~mm} / \mathrm{second}$. Early mitral annular (e'), late diastolic (a'), and annular systolic (s') waves were measured. The proportion of inflow/annular early velocities (E/e') and annular early/annular late velocities (e'/a') were calculated. ${ }^{14)}$ Doppler measurements were recorded twice; first at rest and just after completion of the exercise test. Recording of post-exercise Doppler tracings was concluded in 2-3 minutes following completion of the stress test. The change in E/e' by exercise (E/e' ${ }_{\text {change }}$ ) was calculated as the difference in $\mathrm{E} / \mathrm{e}$ ' by exercise $\left(\mathrm{E} / \mathrm{e}_{\text {change }}=\mathrm{E} / \mathrm{e}_{\text {stress }}{ }_{\text {s. }}-\mathrm{E} / \mathrm{e}_{\text {rest }}\right)$.

Exercise stress test: All patients had undergone treadmill stress testing with a standard Bruce protocol using a Cardioperfect MD System. Blood pressure and heart rate measurements were obtained just before and after the completion of the test. Achievement of at least $85 \%$ of the target heart rate (220-age) and 7 metabolic equivalents (METs) of workload was the aim during the stress test. Patients who were unable to perform this amount of exercise and/or who showed an ischemic response were excluded from our study. As mentioned previously, Doppler echocardiographic variables were recorded just after completion of exercise. ${ }^{15}$

Statistical analysis: Statistical analysis was performed using SPSS 17.0 for Windows (SPSS Inc. Chicago, IL) software.
Comparison of numeric variables between groups was conducted using the $t$-test and nominal variables were compared with the chi-square test. The intraobserver variability of the mean values of Doppler parameters was calculated as percentages and its significance was evaluated with analysis of variance (ANOVA) test.

\section{RESULTS}

A total of 77 individuals (51 in the IR group and 26 controls) were included in this study. As mentioned in the Methods section, patients were free of conservative cardiovascular risk factors. Descriptive variables of the patients are listed in Supplemental Table II. Body mass index $(36.6 \pm 6.0$ versus $\left.32.9 \pm 6.1 \mathrm{~kg} / \mathrm{m}^{2} ; P=0.014\right)$ and waist circumference $(120.8 \pm$ 14.7 versus $108.2 \pm 17.9 \mathrm{~cm} ; P=0.003)$ in the IR group were significantly higher than the controls. Mean glucose levels were similar between the groups, while insulin levels $(18.7 \pm$ 6.8 versus $7.2 \pm 1.3 \mathrm{microU} / \mathrm{mL} ; P=0.000)$ and HOMA-IR index $(4.52 \pm 2.03$ versus $1.65 \pm 0.39 ; P=0.000)$ were significantly higher in the IR group, as expected.

Echocardiographic parameters measured at rest are listed in Supplemental Table III. The left ventricular mass index was similar in both groups. On the other hand, the $\mathrm{E} / \mathrm{A}_{\text {rest }}(P=$ $0.022)$ and $\mathrm{e}^{\prime} / \mathrm{a}_{\text {rest }}(P=0.003)$ values were lower in the IR group. The inflow E/annular e' proportion was not significantly different between the groups $(7.1 \pm 1.6$ versus $6.8 \pm 1.4 ; P=$ $0.522)$.

Doppler parameters measured just after stress testing are listed in Supplemental Table IV. Both groups performed almost the same amounts of exercise $(8.1 \pm 1.1$ versus $8.3 \pm 1.2$ METs; $P=0.754)$. The annular E/A proportion after stress was not significantly different between the groups $(P=0.986)$, whereas the $\mathrm{e}^{\prime} / \mathrm{a}$ 'stress values were smaller $(1.1 \pm 0.4$ versus 1.5 $\pm 0.5 ; P=0.000)$ and $\mathrm{E} / \mathrm{e}_{\text {stress }}$ values were greater $(7.6 \pm 1.8$ versus $6.7 \pm 0.9 ; P=0.007)$ in the IR group compared to the controls; both differences were statistically significant (Supplemental Figures 2, 3). The E/e' value increased by $0.55 \pm$ 1.71 in the IR group, while it decreased by $0.07 \pm 1.08$ in controls by exercise; and this difference was almost significant $(P$ $=0.056)$. The IOV values of the Doppler and TDI Doppler studies were extremely low, as shown in Supplemental Table V.

\section{Discussion}

In this study, diastolic stress echocardiography was performed in a group of otherwise healthy individuals with IR and a control group consisting of healthy individuals without IR. The diastolic function parameters of the patients with IR were more impaired at rest when compared to the controls. Furthermore, diastolic function seemed to be worse after exercise in the IR patients when compared to the controls. The E/e' ratio, an echocardiographic parameter closely related to left ventricular filling pressures, ${ }^{16,17)}$ seemed to rise with exercise in the IR patients; while it did not change significantly in the control group.

Due to increases in heart rate, vasodilatation, contractility, and ventricular filling, cardiac output increases up to 3-fold with exercise in healthy human beings. ${ }^{18)}$ Augmentation of 
preload does not increase ventricular filling pressures in the healthy heart due to increasing elastic recoil and enhanced diastolic relaxation via beta-adrenergic stimulation; both mechanisms provide increased diastolic suction during exercise. This phenomenon is called "diastolic relaxation reserve" and is the primary determinant of exercise capacity in patients with impaired augmentation of heart rate, vasodilation, and contractility with exercise (elderly, patients with hypertension, etc.). ${ }^{19,20)}$

Stress echocardiography is a very useful method for the assessment of coronary artery disease, myocardial viability, and myocardial contractility. Evaluating tissue Doppler recordings in conjunction with the standard protocol increases its feasibility and accuracy. ${ }^{15)}$ Combining mitral early inflow velocity (E) recordings with early mitral annular velocity (e') recordings is very helpful for determining left ventricular (LV) filling pressures. The proportion of mitral inflow velocity to mitral annular velocity (E/e') has been found to be directly associated with LV filling pressures, ${ }^{16)}$ and it is also well correlated with diastolic filling pressures during exercise. ${ }^{21)}$ In a previous diastolic stress echocardiographic study performed by $\mathrm{Ha}$, et al in healthy middle-aged individuals with normal myocardial function, both mitral inflow and annular velocities seemed to rise with exercise. However, no significant change in the E/e' ratio was observed in healthy individuals with normal myocardial function. ${ }^{22)}$ On the other hand, in the two studies performed in patients with heart failure with preserved ejection fraction (HFPEF), the E/e' ratio was significantly higher with exercise in the patient group when compared to the controls and this increase was correlated with exertional dyspnea. ${ }^{20,23)}$ In a stress echocardiography study conducted by Podolec, et al in patients with ischemic heart failure, an increase of the E/e' ratio with exercise was the most important determinant of reduced exercise capacity in this patient population. ${ }^{24)}$ Briefly, an exercise induced increase in the E/e' ratio seems to be a good predictor of reduced ventricular compliance and decreased exercise capacity. ${ }^{25)}$

Due to direct metabolic effects on myocytes, ${ }^{26)}$ and disturbances in the microvascular function ${ }^{27)}$ and autonomic neuropathy ${ }^{28)}$ myocardial dysfunction is a common involvement in T2DM. ${ }^{29)}$ Diastolic dysfunction is the earliest manifestation of diabetic cardiomyopathy and even a small amount is a predictor of a poor prognosis. ${ }^{30,31)}$ In a previous study, diastolic and systolic myocardial dysfunction were detected with tissue Doppler stress echocardiography in asymptomatic T2DM patients, whose traditional echocardiographic signs showed normal diastolic and systolic function. The rise in annular systolic and diastolic waves by exercise was significantly more blunted in the diabetic group. ${ }^{32)}$ In a study conducted in patients who fulfilled at least 2 criteria of metabolic syndrome, exercise E/e' values were inversely correlated with exercise capacity; even in the normal diastolic function range. ${ }^{33)}$ However, a significant proportion of these patients had hypertension unlike our patient group. The study performed by Kosmala, et al in young obese women showed a significant impairment in systolic and diastolic strain rates in the subgroup with insulin resistance. ${ }^{34)}$ However, exercise test was performed only in women older than 35 years old to exclude the presence of coronary artery disease. On the other hand, we performed quantitative stress echocardiography in both male and female individuals and observed exercise induced changes in tissue Doppler parameters. In a very recent study performed by Cadeddu, et al, contractile reserve was shown to be impaired in patients with IR. ${ }^{35)}$ They performed dobutamine stress echocardiography, and showed impaired strain rate increase in IR patients when compared to the controls. ${ }^{35)}$ This is the most similar study to ours and their results are compatible with our study. However, their patient size was smaller than our study (30 IR patients and 20 controls) and some of their patients had controlled HT. Furthermore, patients included in our study were more obese than those in the study by Cadeddu, et al and our findings were primarily focused on diastolic changes with exercise. We may state that the results of our study are complementary to the study performed by Cadeddu, et al.

There are some limitations to our study. The limited patient population restricts us from making more detailed comments and analysis. However, the large list of our exclusion criteria is the main cause of this limited number. It is not easy to find otherwise healthy individuals with IR free of other cardiovascular risk factors in clinical practice. Performing stress echocardiography with a supine bicycle would give more detailed information in every workload of exercise. We performed treadmill exercise testing and were able to evaluate immediate post-exercise changes. Nonetheless, the treadmill exercise stress test is a more physiologic pattern of exercise when compared to the supine bicycle. Another difficulty we had to overcome during this study was the poor echocardiographic image quality. Since we studied an obese population, we observed this problem in spite of the superior harmonic imaging quality of the Vivid 7 ultrasonograph. However, as we stated previously in the exclusion criteria section, we excluded patients with poor image quality. This is another important cause of the limited population size of our study.

In conclusion, myocardial involvement seems to occur in patients with IR, before the appearance of other cardiovascular risk factors. Exercise induced diastolic worsening may be a predictor of reduced compliance and increased ventricular stiffness, although traditional echocardiographic variables showed no structural or functional abnormalities.

\section{Disclosure}

Conflict of interest: None.

\section{REFERENCES}

1. Gill H, Mugo M, Whaley-Connell A, Stump C, Sowers JR. The key role of insulin resistance in the cardiometabolic syndrome. Am J Med Sci 2005; 330: 290-4. (Review)

2. Petersen KF, Shulman GI. Etiology of insulin resistance. Am J Med 2006; 119: S10-6. (Review)

3. Ford ES, Li C, Sattar N. Metabolic syndrome and incident diabetes: current state of the evidence. Diabetes Care 2008; 31: 1898904. (Review)

4. Mottillo S, Filion KB, Genest J, et al. The metabolic syndrome and cardiovascular risk a systematic review and meta-analysis. J Am Coll Cardiol 2010; 56: 1113-32. (Review)

5. Rutter MK, Parise H, Benjamin EJ, et al. Impact of glucose intolerance and insulin resistance on cardiac structure and function: sex-related differences in the Framingham Heart Study. Circulation 2003; 107: 448-54.

6. Ilercil A, Devereux RB, Roman MJ, et al. Associations of insulin levels with left ventricular structure and function in American In- 
dians: the strong heart study. Diabetes 2002; 51: 1543-7.

7. Ingelsson E, Arnlöv J, Lind L, Sundström J. Metabolic syndrome and risk for heart failure in middle-aged men. Heart 2006; 92: 1409-13.

8. Verdecchia P, Reboldi G, Schillaci G, et al. Circulating insulin and insulin growth factor-1 are independent determinants of left ventricular mass and geometry in essential hypertension. Circulation 1999; 100: 1802-7.

9. Witteles RM, Fowler MB. Insulin-resistant cardiomyopathy clinical evidence, mechanisms, and treatment options. J Am Coll Cardiol 2008; 51: 93-102. (Review)

10. Ingelsson E, Sundström J, Arnlöv J, Zethelius B, Lind L. Insulin resistance and risk of congestive heart failure. JAMA 2005; 294: $334-41$.

11. Tuunanen H, Knuuti J. Metabolic remodelling in human heart failure. Cardiovasc Res 2011; 90: 251-7. (Review)

12. Matthews DR, Hosker JP, Rudenski AS, Naylor BA, Treacher DF, Turner RC. Homeostasis model assessment: insulin resistance and beta-cell function from fasting plasma glucose and insulin concentrations in man. Diabetologia 1985; 28: 412-9.

13. Lang RM, Bierig M, Devereux RB, et al. Recommendations for chamber quantification: a report from the American Society of Echocardiography's Guidelines and Standards Committee and the Chamber Quantification Writing Group, developed in conjunction with the European Association of Echocardiography, a branch of the European Society of Cardiology. J Am Soc Echocardiogr 2005; 18: 1440-63.

14. Nagueh SF, Appleton CP, Gillebert TC, et al. Recommendations for the evaluation of left ventricular diastolic function by echocardiography. J Am Soc Echocardiogr 2009; 22: 107-33. (Review)

15. Pellikka PA, Nagueh SF, Elhendy AA, Kuehl CA, Sawada SG. American Society of Echocardiography recommendations for performance, interpretation, and application of stress echocardiography. J Am Soc Echocardiogr 2007; 20: 1021-41.

16. Nagueh SF, Middleton KJ, Kopelen HA, Zoghbi WA, Quiñones MA. Doppler tissue imaging: a noninvasive technique for evaluation of left ventricular relaxation and estimation of filling pressures. J Am Coll Cardiol 1997; 30: 1527-33.

17. Ommen SR, Nishimura RA, Appleton CP, et al. Clinical utility of Doppler echocardiography and tissue Doppler imaging in the estimation of left ventricular filling pressures: A comparative simultaneous Doppler-catheterization study. Circulation 2000; 102: 178894.

18. Higginbotham MB, Morris KG, Williams RS, McHale PA, Coleman RE, Cobb FR. Regulation of stroke volume during submaximal and maximal upright exercise in normal man. Circ Res 1986; 58: 281-91.

19. Cheng CP, Igarashi Y, Little WC. Mechanism of augmented rate of left ventricular filling during exercise. Circ Res 1992; 70: 9-19.

20. Borlaug BA, Jaber WA, Ommen SR, Lam CS, Redfield MM, Nishimura RA. Diastolic relaxation and compliance reserve during dynamic exercise in heart failure with preserved ejection fraction. Heart 2011; 97: 964-9.
21. Burgess MI, Jenkins C, Sharman JE, Marwick TH. Diastolic stress echocardiography: hemodynamic validation and clinical significance of estimation of ventricular filling pressure with exercise. $\mathrm{J}$ Am Coll Cardiol 2006; 47: 1891-900.

22. Ha JW, Lulic F, Bailey KR, et al. Effects of treadmill exercise on mitral inflow and annular velocities in healthy adults. Am J Cardiol 2003; 91: 114-5.

23. Chattopadhyay S, Alamgir MF, Nikitin NP, Rigby AS, Clark AL, Cleland JG. Lack of diastolic reserve in patients with heart failure and normal ejection fraction. Circ Heart Fail 2010; 3: 35-43.

24. Podolec P, Rubis P, Tomkiewicz-Pajak L, Kopeć G, Tracz W. Usefulness of the evaluation of left ventricular diastolic function changes during stress echocardiography in predicting exercise capacity in patients with ischemic heart failure. J Am Soc Echocardiogr 2008; 21: 834-40.

25. Popovic ZB, Griffin B. Diastolic stress testing: a new trick to evaluate the ageing heart. Heart 2010; 96: 906-7.

26. Fang ZY, Schull-Meade R, Downey M, Prins J, Marwick TH. Determinants of subclinical diabetic heart disease. Diabetologia 2005; 48: 394-402.

27. Schleicher ED, Wagner E, Nerlich AG. Increased accumulation of the glycoxidation product $\mathrm{N}$ (epsilon)-(carboxymethyl)lysine in human tissues in diabetes and aging. J Clin Invest 1997; 99: 45768.

28. Sacre JW, Franjic B, Jellis CL, Jenkins C, Coombes JS, Marwick TH. Association of cardiac autonomic neuropathy with subclinical myocardial dysfunction in type 2 diabetes. JACC Cardiovasc Imaging 2010; 3: 1207-15.

29. Fang ZY, Yuda S, Anderson V, Short L, Case C, Marwick TH. Echocardiographic detection of early diabetic myocardial disease. J Am Coll Cardiol 2003; 41: 611-7.

30. Bella JN, Palmieri V, Roman MJ, et al. Mitral ratio of peak early to late diastolic filling velocity as a predictor of mortality in middle-aged and elderly adults: the Strong Heart Study. Circulation 2002; 105: 1928-33.

31. Di Bonito P, Cuomo S, Moio N, et al. Diastolic dysfunction in patients with non-insulin-dependent diabetes mellitus of short duration. Diabet Med 1996; 13: 321-4.

32. Von Bibra H, Thrainsdottir IS, Hansen A, Dounis V, Malmberg K, Rydén L. Tissue Doppler imaging for the detection and quantitation of myocardial dysfunction in patients with type 2 diabetes mellitus. Diab Vasc Dis Res 2005; 2: 24-30.

33. Chockalingam A, Linden MA, Dellsperger KC, Thomas TR. Correlation of Normal Diastolic Cardiac Function With VO in the Metabolic Syndrome. Prev Cardiol 2009; 12: 163-8.

34. Kosmala W, O'Moore-Sullivan TM, Plaksej R, KuliczkowskaPlaksej J, Przewlocka-Kosmala M, Marwick TH. Subclinical impairment of left ventricular function in young obese women: contributions of polycystic ovary disease and insulin resistance. J Clin Endocrinol Metab 2008; 93: 3748-54.

35. Cadeddu C, Nocco S, Piano D, et al. Early impairment of contractility reserve in patients with insulin resistance in comparison with healthy subjects. Cardiovasc Diabetol 2013; 12: 66. 